\title{
AUTOMATIC DETECTION OF LOW-SURFACE BRIGHTNESS GALAXIES ON DIGITAL IMAGES
}

\author{
R.M. SMITH, G.J. PRIVETT, S. PHILLIPPS and J.I. DAVIES \\ Department of Physics \\ University of Wales College of Cardiff \\ P.O. Box 913, Cardiff CF1 3TH \\ Wales, U.K.
}

\section{Introduction}

\begin{abstract}
Algorithms employed to identify and quantify images on digitised images mostly employ contiguous-pixel (e.g. PISA [based on APM software by Irwin \{1985\}]) or median-filtering techniques and thus miss extended low surface-brightness features. Searches for low surface brightness galaxies (LSBGs) have therefore so far concentrated on eye-ball searches of Schmidt survey plates. To overcome the subjectiveness of eyeball searches and eliminate the inherent problems in current object-detection algorithms we have investigated other techniques.
\end{abstract}

\section{Pixel Clustering}

In an attempt to provide some latitude for non-contiguity in the object pixels, a percolation, or friends-of-friends, algorithm has been used. The technique is similar to contiguous-pixel algorithms apart from an extra parameter of maximum object-pixel separation. Any extra objects found by this algorithm, or any that have a much greater area than, from contiguous-pixel algorithms are likely to be LSBGs.

\section{Cross-, Self- and Hybrid-Correlation}

Cross-correlation of the image with an optimised exponential disk template leads to an enhancement of extended objects (Phillipps \& Davies 1991). The set of objects detected is only a weak function of the mask size. The effects of compact objects, bright pixels and cosmic rays are reduced by ignoring the central template pixel and rejecting all pixels greater than $4 \sigma$ above the sky. In self-correlation, all pixels that are equidistant from the central pixel are multiplied by their diametrically opposite pixel. A summation of this product is performed for all pixel pairs within a circular mask and an rms value determined. The value generated, maintaining sign, is ascribed to the output image pixel corresponding to the mask centre. Extended objects will then be enhanced compared to that for compact. Hybrid-correlation is a combination of self- and cross-correlation, weighting each pixel pair in the self-correlation by the exponential mask. In 
all the correlation techniques, LSBGs are found by their greater enhancement over their original image.

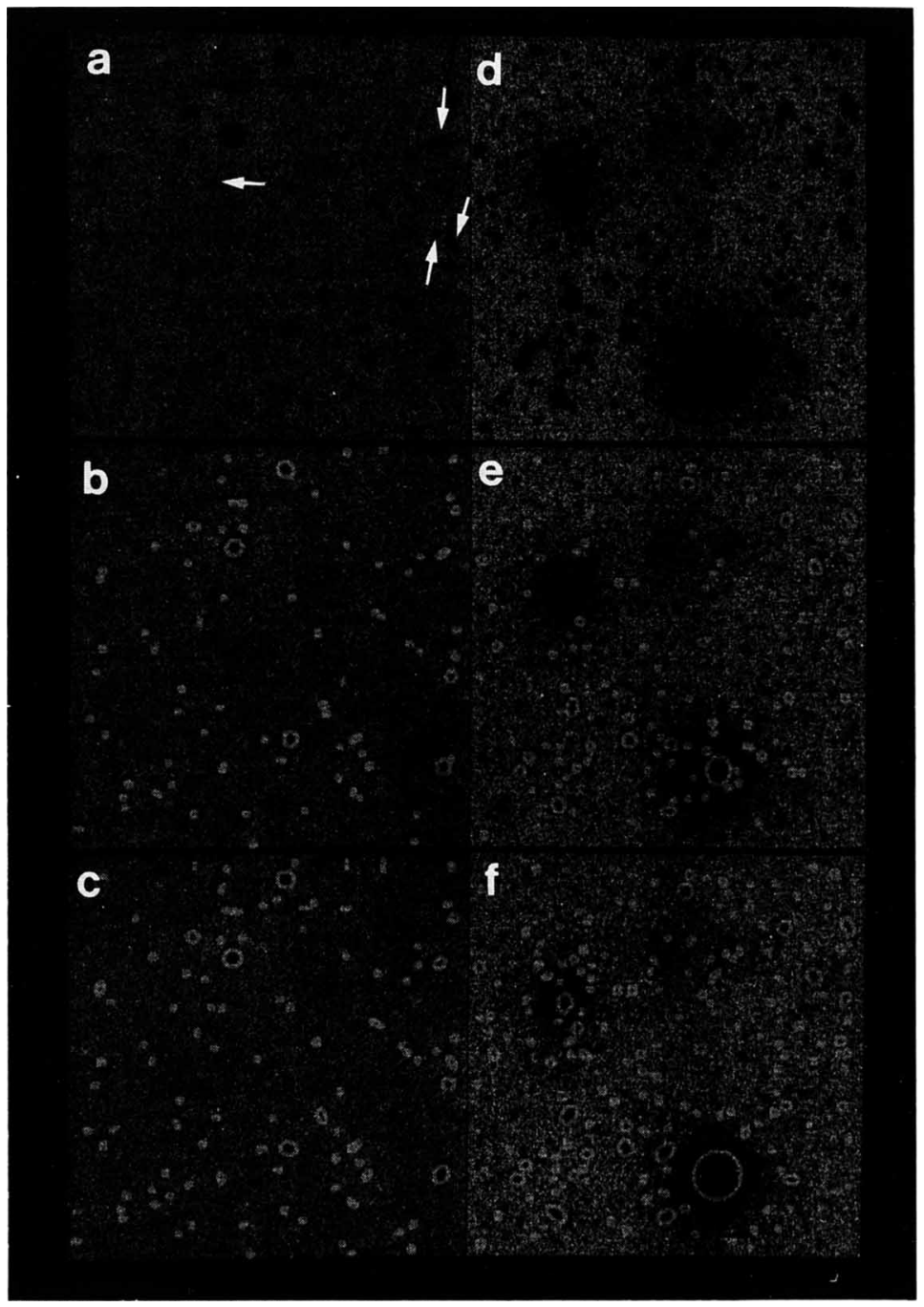

Figure 1. Results of the application of PISA and pixel-clustering object detection algorithms. (a) An APM scan of part of the Fornax cluster with known LSBGs marked, (b) application of PISA to (a), and (c) application of pixel-clustering method to (a). (d), (e) and (f) show the same techniques applied to simulated CCD image with a 'normal' galaxy population added, together with LSBGs of varying scalelengths and central surface-brightnesses. 


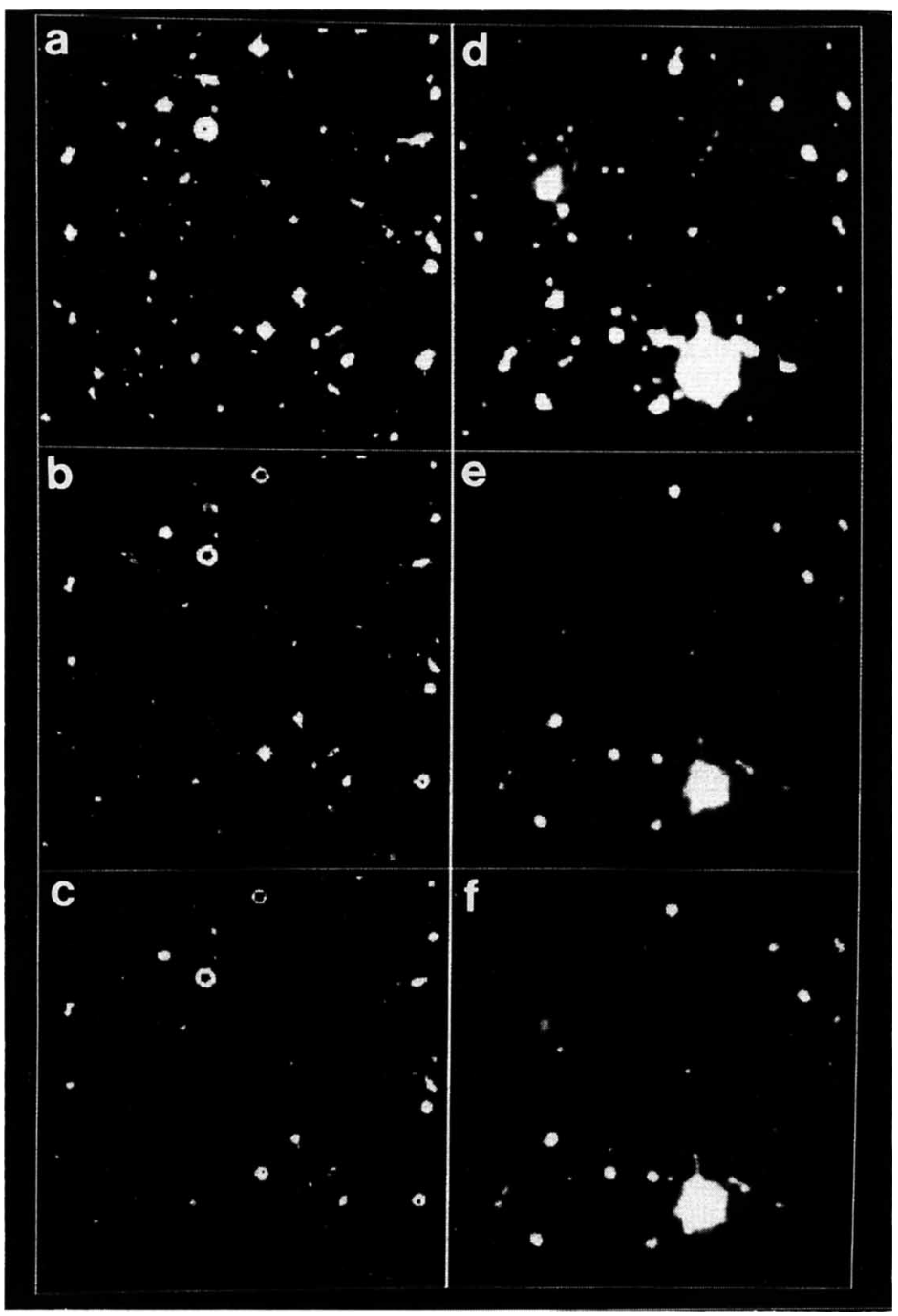

Figure 2. As Fig. 1 except the techniques employed are, top to bottom, cross-correlation, self-correlation, and hybrid-correlation. 


\section{Results and Discussion}

The results of these methods applied to the two test frames are shown in Figs. 1 and 2. All techniques successfully detect the majority of the LSBGs, as shown in Table 1. Hybridcorrelation gives the greatest contrast enhancement for the correlation techniques. PISA only detects 2 objects (rising to 3 if the data are smoothed before application of the algorithm). These new methods therefore show great promise in the search for LSBGs using digital images.

Table 1. Positions of detected LSBGs in the simulated CCD test frame. For comparison between the pixel-clustering and PISA algorithms, the total number of pixels in each object is also listed. Positions of objects that are only marginally detected are marked by an asterisk.

\begin{tabular}{llllll}
\hline True & PISA & Cluster & Cross & Self & Hybrid \\
\hline \hline 810,630 & $806,626: 3252$ & $797,633: 11355$ & 810,629 & 809,626 & 810,629 \\
370,240 & - & 370,242 & 370,239 & 371,239 & 370,240 \\
250,490 & - & 261,467 & 259,490 & 250,490 & 250,488 \\
250,650 & - & 245,649 & 252,647 & 250,649 & 250,649 \\
70,470 & $67,470: 96$ & $67,469: 188$ & 69,470 & 68,469 & 68,469 \\
900,80 & - & 899,79 & 899,79 & 899,78 & 899,77 \\
840,832 & - & - & - & $838,831^{*}$ & $833,830^{*}$ \\
450,390 & - & - & $451,391^{*}$ & $447,390^{*}$ & $449,385^{*}$ \\
640,580 & - & 643,580 & 641,579 & 640,579 & 640,579 \\
\hline
\end{tabular}

\section{References}

Irwin, M.J., 1985. Mon. Not. $R$. astron. Soc., 214, 575.

Phillipps, S. and Davies, J.I., 1991. Mon. Not. R. astron. Soc., 251, 105. 